\title{
Pars Plana Vitrectomy for Full-Thickness Macular Holes in Patients with Proliferative Diabetic Retinopathy and Active Fibrovascular Proliferation
}

This article was published in the following Dove Press journal:

Clinical Ophthalmology

\section{Mushfig Karimov Lala Akhundova (iD) \\ Tarlan Aliyeva}

Department of Diabetic Eye Diseases, National Centre of Ophthalmology Named After Acad. Zarifa Aliyeva, Baku, Azerbaijan
Correspondence: Lala Akhundova Department of Diabetic Eye Diseases, National Centre of Ophthalmology Named After Acad. Zarifa Aliyeva, Baku, Azerbaijan

Tel +994 50-3565।8।

Email akhundova.lale@gmail.com
Purpose of the Study: To study preoperative and postoperative optical coherence tomography features and the outcomes of pars plana vitrectomy (PPV) with internal limiting membrane (ILM) peeling for full-thickness macular holes (MHs) associated with proliferative diabetic retinopathy.

Methods: The data from 14 eyes of 11 patients with full-thickness macular holes and active diabetic fibrovascular proliferation (FVP) with/without tractional retinal detachment who underwent pars plana vitrectomy and standard ILM peeling at Zarifa Aliyeva National Ophthalmology Centre in Baku were analysed. Bilateral surgery for diabetic MHs was performed in $27.3 \%$ of patients. The minimum follow-up duration was 6 months.

Results: All eyes (100\%) achieved type 1 macular hole closure, with residual macular subretinal fluid (SRF) present in 13 cases after surgery (92.9\%). The SRF resolved gradually without any interventions. The incidence of SRF was $92.9 \%$ at 1 month, $85.7 \%$ at 3 months, $50 \%$ at 6 months, and $14.3 \%$ at 9 months (Cochran's $Q$ test, $\chi 2(4)=37.44, p<0.001)$. Visual acuity improved after surgery in 13 cases $(92.9 \%)$, with a mean difference of $0.75 \pm 0.71$ LogMAR between the preoperative and final values, $\mathrm{r}_{\mathrm{s}}=0.608,95 \%$ BCa CI [0.037-0.969], $p=0.021$.

Conclusion: The standard ILM peeling technique for full-thickness MHs in eyes with active diabetic fibrovascular proliferation can help restore the anatomy and function of the macula in diabetic patients. Close follow-up is important for unilateral cases. Residual SRF in the perifoveal area is detectable by OCT after surgery in patients with macular holes associated with diabetic tractional retinal detachment, and it usually resorbs gradually without any interventions.

Keywords: pars plana vitrectomy with ILM peeling, tractional retinal detachment, residual subretinal fluid, optical coherence tomography, macular hole, proliferative diabetic retinopathy

\section{Plain Language Summary What Was Known Before}

A full-thickness macular hole is a rare complication of diabetic retinopathy that is responsible for only $1.2 \%$ of the total number of vitrectomies performed for complications of diabetic retinopathy.

\section{What This Study Adds}

The frequent occurrence of bilateral cases indicates that the fellow eyes need to be followed up closely and that active fibrovascular proliferation is a risk factor for the development of 
macular holes.Vitrectomy with standard ILM peeling is an effective treatment for closure of macular holes in eyes with active diabetic fibrovascular proliferation.Type 1 macular hole closure after the single surgery (not including phacoemulsification and planned silicone oil removal) was achieved in all cases (100\%). Residual subretinal fluid is observed after surgery in most cases $(92.9 \%)$ in contrast to idiopathic macular holes and it usually resorbs gradually without any interventions. The characteristic course of subretinal fluid resorption after surgery: the fovea attached first and the subretinal fluid gradually resolved in the perifoveal area.

\section{Introduction}

Diabetic retinopathy is one of the major causes of blindness and low vision among working-age population in developed countries. ${ }^{1}$ Proliferative diabetic retinopathy (PDR) is considered an advanced stage of the disease; many cases require surgical intervention and have a poor visual prognosis. ${ }^{2}$ Pars plana vitrectomy is indicated for various complications of PDR, such as non-resolving vitreous haemorrhage, tractional retinal detachment, and vitreomacular traction. ${ }^{3}$ In rare cases, active fibrovascular proliferation (FVP) in PDR can be complicated with a full-thickness macular hole $(\mathrm{MH})$, which has a worse prognosis than idiopathic $\mathrm{MH}^{4-11}$ Most of the reports in the literature concerning the management of diabetic retinopathy and $\mathrm{MH}$ were conducted in the pre-OCT era, and detailed preoperative and postoperative spectral domain OCT analyses of diabetic MHs were conducted in only a few recent studies. ${ }^{10-12}$

Internal limiting membrane (ILM) peeling was first used for the treatment of idiopathic macular holes. ${ }^{13}$ The development of various surgical adjuvants, in particular, vital dyes for vitreoretinal surgery, has widened the surgical indications for this treatment and led to improved outcomes of ILM peeling for various retinal disorders, including diabetic macular holes. ${ }^{3,11}$ As novel surgical techniques of ILM peeling, such as the inverted ILM flap, the free ILM flap, fovea-sparing ILM peeling, and macular hole hydrodissection, were developed to further improve the surgical treatment results of idiopathic MHs, the usefulness of the standard ILM peeling technique in treating more complicated cases, such as diabetic MHs, remains unknown. ${ }^{11,14,15}$ This study also aimed to determine whether the standard ILM peeling technique for fullthickness MHs in eyes with active diabetic fibrovascular proliferation is sufficient for anatomical and functional success.

\section{Materials and Methods}

In this retrospective case study, out of 970 consecutive cases of various complications of PDR for which pars plana vitrectomy was performed from July 2009 to June 2019, 14 eyes of 11 patients $(1,44 \%)$ were identified as having fullthickness macular holes and active diabetic FVP with/without tractional retinal detachment and were included in the study. The study design was approved by the ethics committee of the National Ophthalmology Centre named acad. Zarifa Aliyeva according to the criteria set by the declaration of Helsinki and each subject signed an informed consent before participating to the study as well as consent to publish was obtained from all participants. The indication for surgery was $\mathrm{MH}$ in 14 cases (combined with tractional retinal detachment in 2 cases). The eyes met the inclusion criteria if the macular holes were diagnosed preoperatively by spectral domain OCT, underwent pars plana vitrectomy and standard ILM peeling, and were followed up for a minimum of 6 months. Patients with lamellar holes, pseudoholes, secondary macular holes after previous vitrectomy, and macular holes related to trauma or another aetiology and those without concomitant proliferative diabetic changes were excluded from the study.

The routine ocular examinations performed at the preoperative and postoperative visits included a BCVA examination, slit-lamp biomicroscopy with a $90 \mathrm{D}$ lens, fundus photography, ultrasonography, and spectral domain OCT. Spectral domain optical coherence tomography (SD OCT) was performed for all cases with the Cirrus-HD OCT device (Carl Zeiss Meditec, USA) and 2 protocols - a macular cube of $512 \times 128$ with retinal thickness measurements in 9 zones and a 5-line HD raster. OCT measurements of the macular hole, the height of the detachment, the vitreoretinal relationship and structural changes were recorded. The automated central foveal thickness (Automated-CFT) corresponded to the central $1000 \mu \mathrm{m}$ diameter and was calculated for 9 zones. The size of the macular hole, height of the subretinal fluid and central foveal thickness (Manual-CFT) were measured manually with callipers on the SD OCT machine. The photoreceptor ellipsoid layer (EZ) and external limiting membrane (ELM) layer integrities were evaluated within the area measuring $1000 \mu \mathrm{m}$ in diameter in the central fovea. We revise the video records of the surgery to recheck the status of the posterior hyaloid.

Fibrovascular proliferation was considered active if the new blood vessels were noted on more than $1 / 3$ of the surface, minimally active if the new blood vessels were 
noted on less than $1 / 3$ of the surface, and non-active if the active vessels were absent or there were mainly sclerosed vessels.

\section{The Surgical Technique}

For standard mini-invasive 23-gauge vitrectomy, the Accurus $800 \mathrm{CS}$ or Constellation (Alcon, USA) surgical systems were used with the BIOM $3 \mathrm{~m} / 4 \mathrm{~m}$ fundus visualization system (Oculus, Germany). If a combined surgery was planned, phacoemulsification of the cataract and implantation of the IOL into the capsular bag were performed at the beginning of the surgery. After core vitrectomy, the posterior hyaloid was stained with triamcinolone and identified, anterior-posterior tractions were removed, and then, all fibrovascular tissues at the posterior pole were removed as thoroughly as possible. In most cases, epiretinal fibrovascular membranes were removed with the vitrectomy probe only, and microscissors were rarely used. The internal limiting membrane was removed in a circular fashion of 360 degree around the macular hole after staining with trypan blue or brilliant blue $\mathrm{G}$ dye (BBG) with disposable ILM forceps (Alcon, USA) in a standard manner approximately no less than 2-disc diameters from the fovea. For internal tamponade, long-acting gas $\left(14 \% \mathrm{C}_{3} \mathrm{~F}_{8}\right.$ /air mixture) or silicone oil (1000 centistokes) was used at the end of surgery.

\section{Statistical Analysis}

All statistical tests were performed with IBM SPSS Statistics software, version 20.0 (IBM Corp., Armonk, NY, USA). BCVA values were converted to LogMAR (logarithm of the minimum angle of resolution) values for statistical processing. The means \pm standard deviations were calculated for the quantitative data. The Mann-Whitney $U$-test was performed to compare the SRF resorption time across groups. Cochran's Q test was used to compare the incidence of SRF across time points. Friedman's ANOVA was used to compare visual acuity across visits. Spearman correlation was used to correlate different nonparametric variables, and a biserial correlation test was performed in cases of continuous dichotomy. A multiple regression analysis was used to predict the final BCVA (preoperative BCVA, time since diagnosis of the macular hole, the size of the macular hole, height of the SRF, vitreous haemorrhage, hyaloid status, panretinal photocoagulation, cystoid macular oedema, the type of tamponade, the duration of the postoperative SRF, ELM/EZ layers changes after surgery). Logistic regression analysis was conducted with significant preoperative individual or multiple factors (the time since diagnosis of the macular hole, the size of the macular hole, the height of the SRF, vitreous haemorrhage, hyaloid status, panretinal photocoagulation, cystoid macular oedema, the type of tamponade) to predict long-standing SRF in postoperative time.

\section{Results}

We analysed the data from 14 eyes of 11 patients with fullthickness macular holes and diabetic active fibrovascular proliferation who underwent pars plana vitrectomy and standard ILM peeling at Zarifa Aliyeva National Ophthalmology Centre in Baku. Three patients underwent surgery in both eyes. Another 2 patients had MHs with duration of more than 1 month in the fellow eye and old tractional-rhegmatogenous detachment, and surgery was not recommended due to a poor functional prognosis. All patients had type 2 diabetes with a mean duration of diabetes of $8.71 \pm 3.79$ years (range, 5-18 years). The mean age of the patients was $55.86 \pm 57.2$ years (range, 48-65 years). The demographic and clinical characteristics of the patients are presented in Table 1.

Preoperatively, extensive epiretinal membranes were noted in all eyes (Figure 1, top). In the preoperative OCT examination, subretinal fluid (SRF) in the macula was noted in 13 eyes (92.9\%) (Figure 2, top), and the mean height of the macular detachment was $595.0 \pm 371.33 \mu \mathrm{m}$ (range, 0-1200 $\mu \mathrm{m}$ ). The average $\mathrm{MH}$ diameter was 681.36 $\pm 157.16 \mu \mathrm{m}$ (range, 414.0-869.0 $\mu \mathrm{m}$ ). Cystoid macular oedema was noted in 12 eyes (85.7\%). Anti VEGF treatment done for 1 patient preoperatively. Incomplete posterior vitreous detachment (PVD) with focal adhesion at the posterior pole was detected in 1 eye $(7.1 \%)$, less than 2 quadrants of adhesion of posterior hyaloid was noted in 9 eyes $(64.3 \%)$, and more than 2 quadrants of adhesion was noted in 4 eyes $(28.6 \%)$. Broad adhesions of FVP at the posterior pole were noted in all cases. Tractional retinal detachment (TRD) within the arcade was found in 2 cases (14.3\%), and TRD within and outside the arcade but posterior to the equator was found in 12 cases $(85.7 \%)$.

No major complications were recorded during surgery in any eye. Iatrogenic breaks were observed in 1 case $(7.14 \%)$ with additional drainage of the SRF. In 2 patients $(14.3 \%)$ with blind fellow eyes, silicone oil was used as a tamponade agent and was removed uneventfully after 3 and 12 months. In all other cases (12 eyes, 85.7\%), a long- 


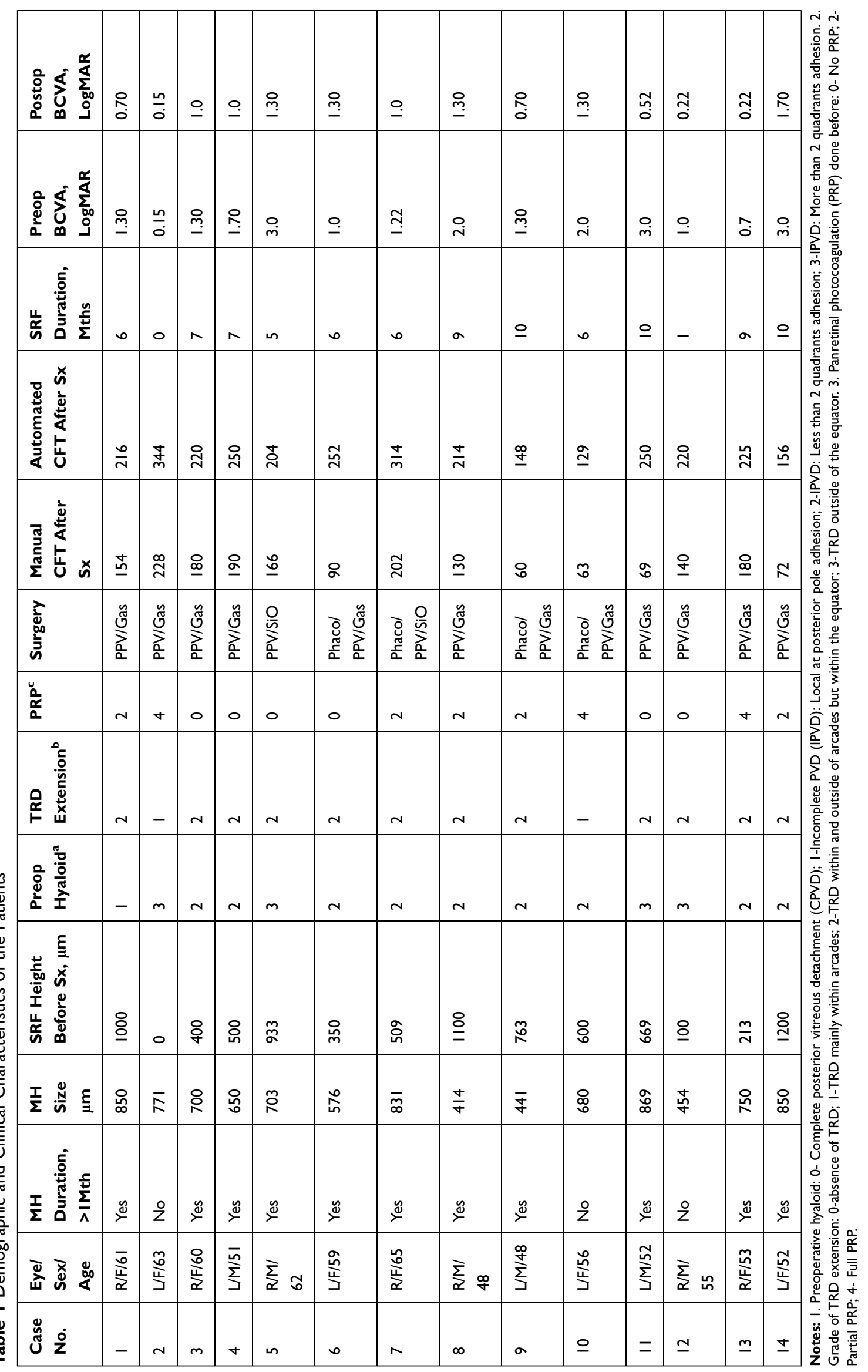




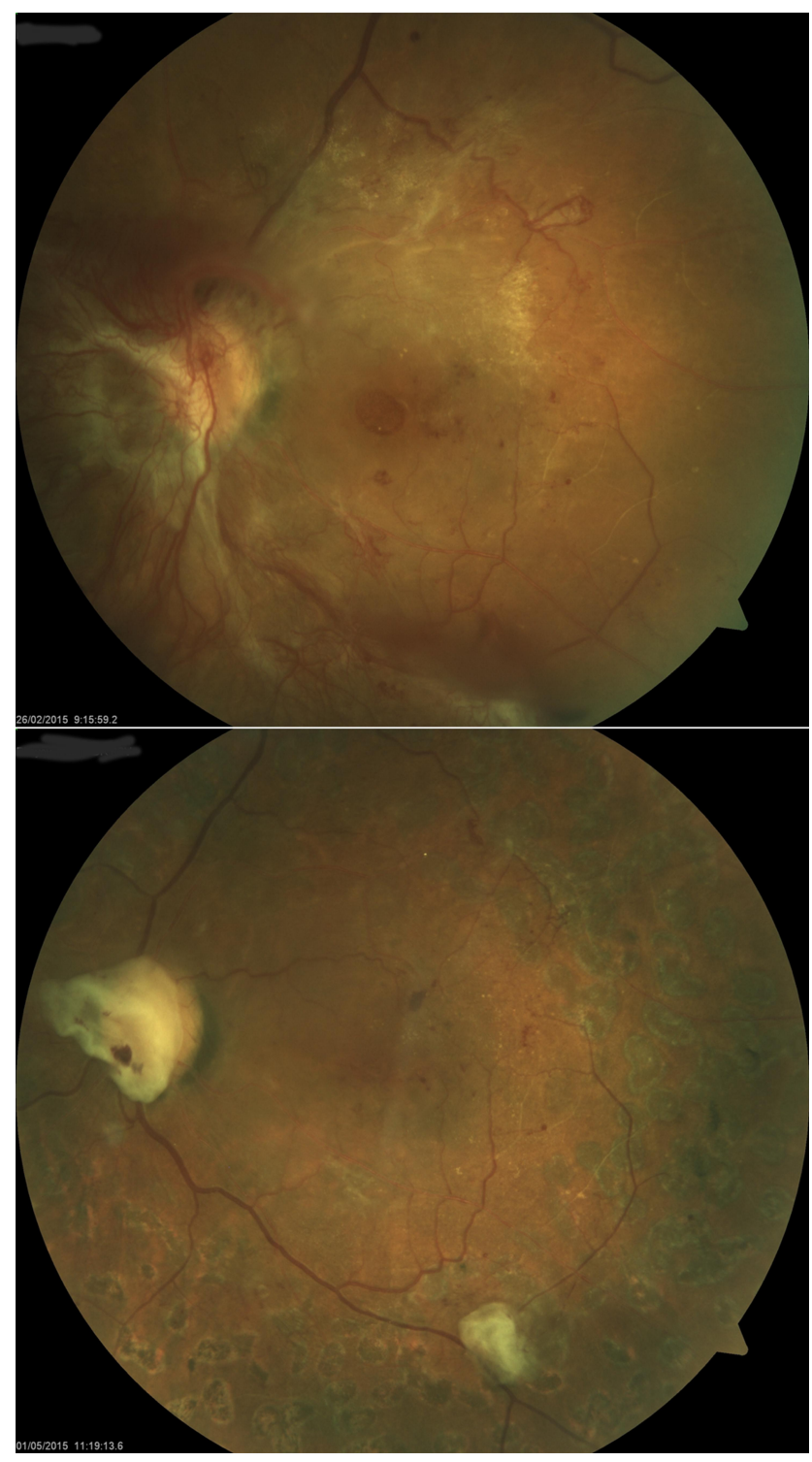

Figure I Preoperative appearance (top) of the fundus of the left eye in a 52 yearsold diabetic patient with a full-thickness macular hole. Proliferative membranes are seen with active neovascularization. After vitrectomy (bottom) with ILM peeling, retina is quiet, membranes are removed and macular hole is closed.

acting air-gas mixture was injected at the end of surgery.

Residual vitreous cavity haemorrhage was noted in 2 patients $(14.3 \%)$, with complete resorption at 6 weeks after surgery.

The mean follow-up time was $21.79 \pm 20.41$ months (range, 7-83 months). Type 1 macular hole closure after the single surgery (not including phacoemulsification and planned silicone oil removal) was achieved in all cases $(100 \%)$ (Figure 1). Residual macular SRF was noted in the OCT examination in 13 cases after surgery (92.9\%) (Figure 2), and the SRF resolved slowly without any interventions after a mean duration of $6.57 \pm 3.11$ months

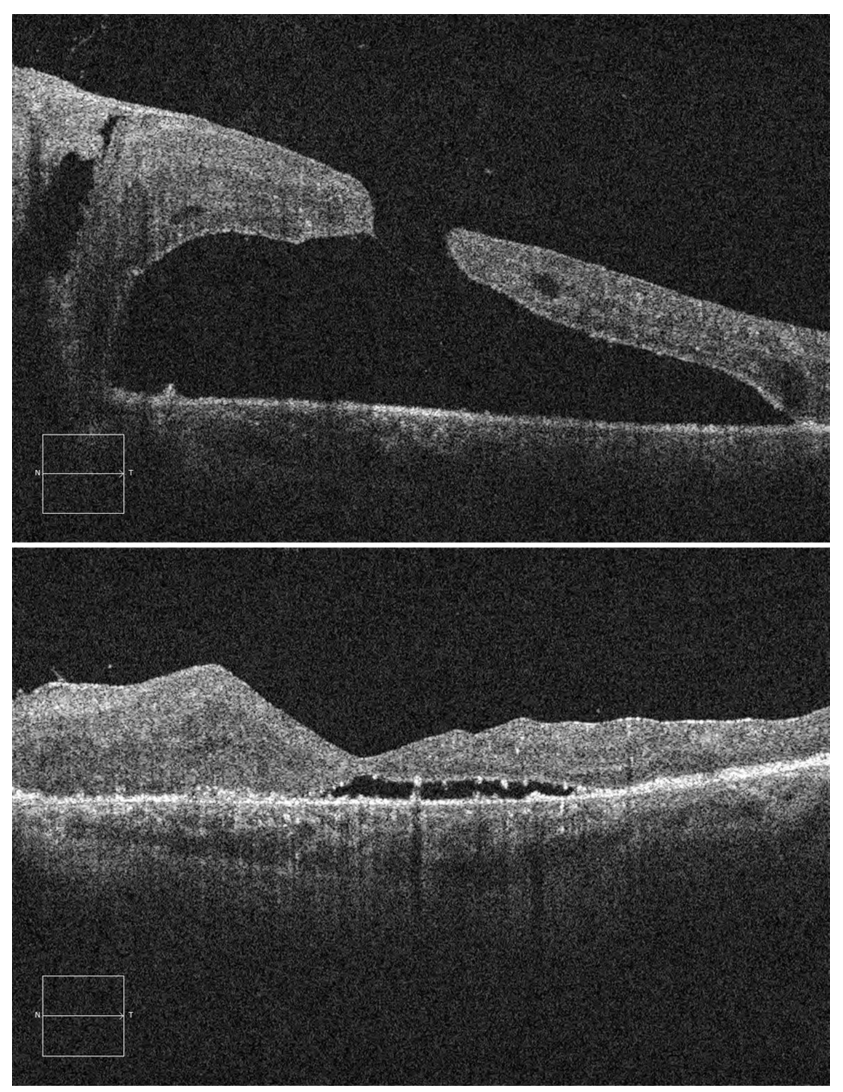

Figure 2 OCT appearance of the patient in the Fig. I. Preoperative OCT (top) shows a full-thickness retinal defect with macular detachment. Two months after surgery (bottom), macular hole is closed, but residual SRF is noted perifoveally.

(range, 1-10 months) (Figure 3). The incidence of SRF was $92.9 \%$ at 1 month, $85.7 \%$ at 3 months, $50 \%$ at 6 months, and $14.3 \%$ at 9 months (Cochran's Q test, $\chi^{2}$ (4) $=37.44, p<0.001)$. At 3 months after surgery, residual SRF was significantly related to the LogMAR BCVA, $r_{\mathrm{b}}=$ $-0.88, p=0.034$. The SRF resorption time differed across groups stratified by the MH duration, type of endotamponade, MH size, and height of the SRF at the preoperative examination, but only the difference between the $\mathrm{MH}$ with duration of 1 month or less and $\mathrm{MH}$ with duration of more than 1 month was statistically significant (Table 2). The final manual CFT was $137.43 \pm 57.29 \mu \mathrm{m}$ (range, 60-228 $\mu \mathrm{m}$ ), and the automated CFT was $224.429 \pm 58.61 \mu \mathrm{m}$ (range, 129-344 $\mu \mathrm{m}$ ). Manual CFT was significantly related to automated CFT, with a mean difference of $86.999 \mu \mathrm{m}, \mathrm{r}_{\mathrm{s}}=0.67,95 \%$ BCa CI [0.138,0.978], $p=0.009$. Manual CFT $\left(r_{\mathrm{s}}=-0.304, p=0.291\right)$ and automated CFT $\left(r_{\mathrm{s}}\right.$ $=-0.420, p=0.135)$ were not related to the final LogMAR BCVA. The ELM/EZ layers were disorganized at the last follow-up in 9 cases $(64.3 \%)$, and this factor had a significant negative correlation with the final LogMAR BCVA, $r_{\mathrm{b}}=-.0876, p=0.008$. 

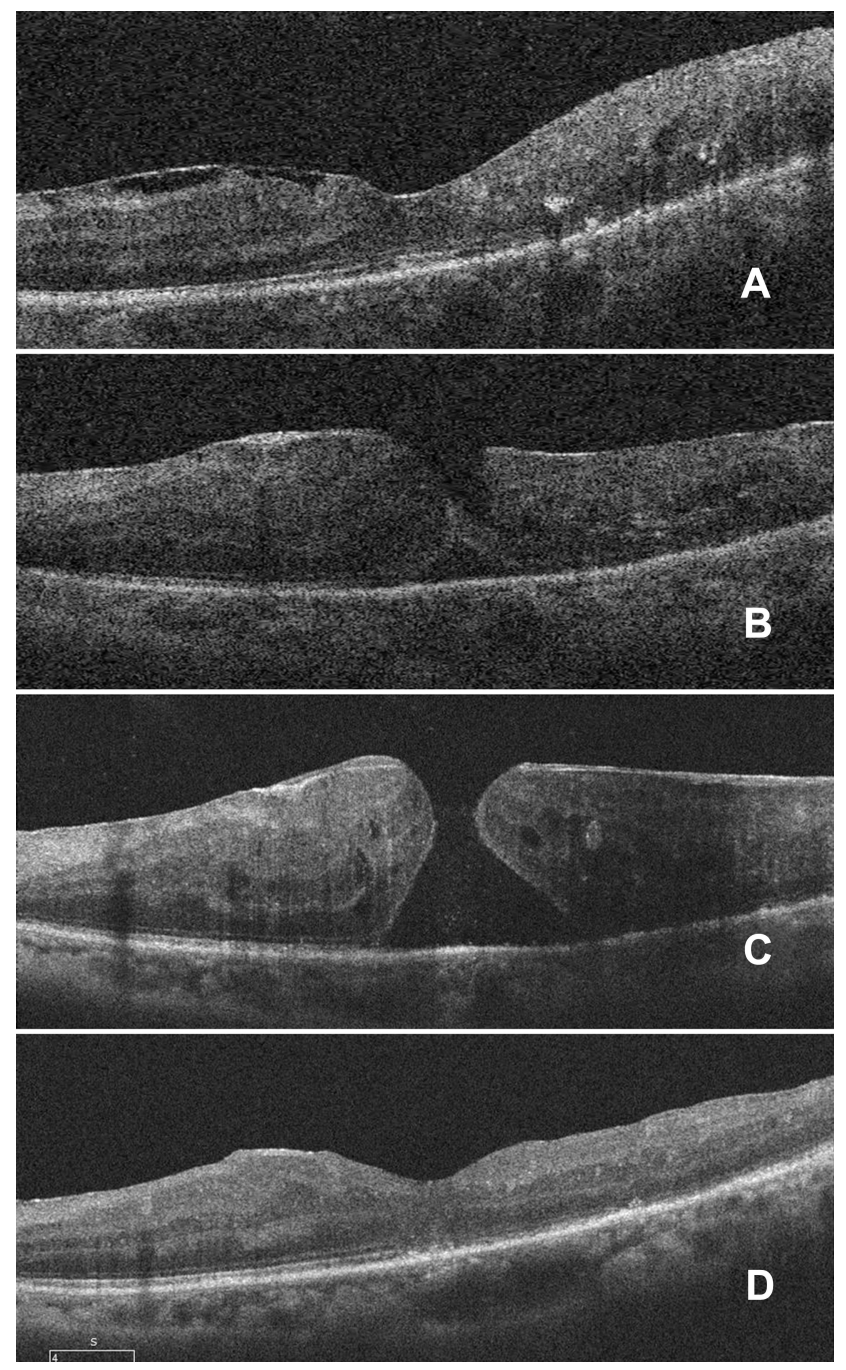

Figure 3 Development of the full-thickness macular hole in the left eye of a 63 years-old diabetic patient. (A) Fovea is intact, but epimacular membranes and tangential traction are noted (B) Seven months later, macular oedema and separation of the internal and external retinal layers developed. (C) Four months later, a full-thickness macular hole is formed. (D) Three months after surgery, MH is closed, mild disorganization of EZ is noted.

The preoperative visual acuity was $1.62 \pm 0.89$ LogMAR (range, 0.15-3.0 LogMAR), and it continued to improve postoperatively for 6 months to $0.90 \pm 0.47$ LogMAR (range, 0.15-1.70); there was a slight decline at 9 months to $1.0 \pm 0.46 \operatorname{LogMAR}$ (range, 0.30-1.70), $X^{2}$ (3) $=15.26, p=0.002$ (Friedman's ANOVA test). In general, visual acuity improved after surgery in 13 cases $(92.9 \%)$, with a mean difference of $0.75 \pm 0.71$ LogMAR between the preoperative and final visual acuity values, $r_{\mathrm{s}}=0.608$, 95\% BCa CI [0.037-0.969], $p=0.021$. The multiple regression analysis revealed that only the preoperative SRF height significantly predicted the final BCVA $(F=5.271$, $\left.R^{2}=0.489, p=0.008\right)$. Logistic regression analysis did not reveal any individual or multiple factors that were significant in predicting the duration of SRF postoperatively. All the statistically nonsignificant factors included in the regression analysis are not reported here.

\section{Discussion}

A full-thickness macular hole is an uncommon complication of diabetic retinopathy that is responsible for only $1.2 \%$ of the total number of vitrectomies performed for complications of diabetic retinopathy. ${ }^{14}$ In the present study, the primary indication for vitrectomy was $\mathrm{MHs}$ for $1.44 \%$ (14 eyes) of 970 eyes that underwent surgery for PDR complications. Out of 11 patients, three underwent bilateral surgery for diabetic MHs (27.3\% of cases), but of the remaining 8 patients, 2 had between the $\mathrm{MH}$ with duration of more than 1 month with old tractionalrhegmatogenous retinal detachment in the fellow eyes that were not treated due to poor functional prognosis. The frequent occurrence of bilateral cases indicates that the fellow eyes need to be followed up closely and that active FVP is a risk factor for the development of MHs.

The mechanism of MH development in DR is considered different from idiopathic macular holes and is suggested to be due to tangential traction from fibrovascular proliferation on the oedematous and ischaemic diabetic macula. ${ }^{4,8,9,11,16}$ In DR, the large amount of perimacular tangential traction exerted by fibrovascular membranes on the fovea is responsible for the development of $\mathrm{MHs}$, which are usually associated with a higher degree of retinal elevation than are idiopathic MHs. ${ }^{10} \mathrm{We}$ were able to trace the development of a full-thickness $\mathrm{MH}$ caused by active FVP, starting with the separation of internal and external layers (Figure 3). We hypothesize that active fibrovascular proliferation, especially with broad adhesion, exerts more traction than does minimally active or non-active tissue. The extent of fibrovascular proliferation is also an important factor for the progression of macular holes, as broad adhesions posterior to the equator were detected frequently in our study.

Some authors suppose that long-standing macular oedema with tractional forces can easily cause MHs, with oedematous macula in particular being vulnerable to vitreous traction. ${ }^{6,17}$ Cystoid macular edema, which may be an additional causative factor in the development of macular holes, was found in $85.7 \%$ of our cases, and the epiretinal membrane was identified in all cases preoperatively. Since we did not observe cystic changes in all cases, we suggest that tangential traction is the most important factor for $\mathrm{MH}$ development. An incomplete 
Table 2 The Mean SRF Resorption Time in Different groups ${ }^{\mathrm{a}}$

\begin{tabular}{|c|c|c|c|c|c|}
\hline - & - & Number of Cases & Test Statistic-U & Months Mdn (IQR) & $\mathbf{P}^{\mathbf{a}}$ \\
\hline All cases & _ & 14 & - & $6.5(3.5)$ & - \\
\hline MH duration & $\begin{array}{l}\leq 1 \text { month } \\
>I \text { month }\end{array}$ & $\begin{array}{l}2 \\
12\end{array}$ & 24 & $\begin{array}{l}0.5(-) \\
7.0(3.8)\end{array}$ & 0.022 \\
\hline Endotamponade & $\begin{array}{l}\text { Gas } \\
\text { Silicone oil }\end{array}$ & $\begin{array}{l}12 \\
2\end{array}$ & 5.5 & $\begin{array}{l}7.0(3.8) \\
5.5(-)\end{array}$ & 0.264 \\
\hline Preoperative SRF height & $\begin{array}{l}\leq 500 \mu \mathrm{m} \\
>500 \mu \mathrm{m}\end{array}$ & $\begin{array}{l}6 \\
8\end{array}$ & 33 & $\begin{array}{l}6.5(6.8) \\
7.5(4.0)\end{array}$ & 0.282 \\
\hline Preoperative $\mathrm{MH}$ size & $\begin{array}{l}\leq 700 \mu \mathrm{m} \\
>700 \mu \mathrm{m}\end{array}$ & $\begin{array}{l}7 \\
7\end{array}$ & 23.5 & $\begin{array}{l}7.0(3.0) \\
6.0(5.0)\end{array}$ & 0.902 \\
\hline
\end{tabular}

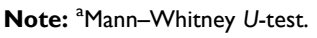

PVD in cases of PDR may also cause additional vitreoretinal traction and may be a predisposing factor for $\mathrm{MH}$ formation; incomplete PVD was noted in all cases with different degrees of adhesion. Therefore, we agree with the previous findings showing that if there is no central posterior vitreous detachment, macular holes can be caused by tangential traction in oedematous and ischaemic fovea. ${ }^{14}$

MHs associated with PDR were first mentioned by Amemiya et al (1978), who described 3 cases of MHs associated with fibrous proliferation due to PDR. ${ }^{6}$ Due to the technological limitations at the time, one case of a $\mathrm{MH}$ was treated with photocoagulation, and the other two cases were treated with only observation, and the visual acuity of all three patients deteriorated. Later, Flynn (1994) described 2 cases of MHs associated with PDR, which were successfully treated with vitrectomy and gas tamponade only; there were minor improvements in visual acuity. ${ }^{9}$ In later reports, the introduction of ILM peeling significantly improved the anatomical results of surgery for diabetic MHs. ${ }^{5,8,11,16}$

In the pre-OCT era, some authors used endodrainage to differentiate full-thickness MHs from partial-thickness MHs. ${ }^{14}$ Yeh et al conducted the largest case series to date that has been reported in the literature, and the results were confirmed with time-domain OCT. $^{8}$ In that series, ILM peeling was applied selectively during the surgery. After the release of diabetic fibrovascular tissue with or without ILM peeling, the MH was closed in $82.6 \%$ of the cases. Though the authors did not find a statistically significant difference between the groups who did and did not undergo ILM peeling, all the cases of open MHs were in eyes that were not treated with ILM peeling. The recent case series presented by S. Chen (2018) reported different
ILM peeling techniques, such as the inverted internal limiting membrane (ILM) flap technique, inverted ILM insertion and free ILM flaps, being performed in 10 patients, with successful results. ${ }^{11}$ Other nonstandard ILM peeling techniques have introduced several challenges in treating diabetic eyes due to the fragility of the flap in ischaemic diabetic macula and the difficulty in maintaining the position of the flap during fluid-air exchange, especially in detached macula. An advantage of the standard ILM peeling technique is its simplicity.

In our study, standard ILM peeling was performed in all cases, and the single surgery anatomical success rate was $100 \%$, with type 1 closure of the MH being achieved in all cases, despite the MHs being large (average size, $681.36 \pm 157.16 \mu \mathrm{m}$, range, $414.0-869.0 \mu \mathrm{m}$ ) and macular detachment being present (average height, 595.0 \pm 371.33 $\mu \mathrm{m}$, range, $0-1200 \mu \mathrm{m})$. One of the explanations for $\mathrm{MH}$ closure in such scenarios may be the greater stretch of retinal tissue in relatively high macular detachment. ${ }^{8}$ After the release of traction, the stretched tissue may be redundant enough to close the holes after the retinal detachment settles. Another factor may be the proliferative environment, which can promote the closing of the gap. ${ }^{8}$ A technique recently recommended for the closure of persistent, chronic, and large MHs is hydrodissection of the macula. ${ }^{15}$ The authors concluded that hydration of the retina at the edges of the MH causes lysis of retina-RPE adhesions and increases the tissue volume and the compliance of the retina. The last presumption might also be valid for our cases since all but 1 patient had diabetic tractional detachment without retina-RPE adhesions at the $\mathrm{MH}$ edges. After successful surgery for MH cases with macular detachment, slow-resolving residual SRF 
was commonly found. In previous studies, the slow absorption of SRF after vitrectomy without drainage in eyes with macular tractional retinal detachment has been reported.2,18] The residual SRF after TRD surgery is explained by the chronic nature of the fluid in TRD. Internal drainage of the SRF was not helpful in all cases and was recommended only in cases of intraoperative breaks. The SRF usually resolved spontaneously, with improvements in visual acuity. In the present study, the mean duration that SRF existed after surgery was 6.57 \pm 3.11 months, with an incidence of SRF of $-92.9 \%$ at 1 month, $85.7 \%$ at 3 months and $14.3 \%$ at 9 months. The SRF resolved gradually without any interventions in all cases. At 3 months after surgery, residual SRF was found to be significantly related to the LogMAR BCVA, $r_{\mathrm{b}}=$ $-0.88, p=0.034$. In the present study, a correlation between the macular hole duration and SRF resorption ( $p=0.022$ ) was found, which confirmed the chronic nature of the submacular fluid. An interesting finding of the study was that the SRF height was significantly correlated with the final BCVA $\left(F=5.271, R^{2}=0.489, p=0.008\right)$. Another interesting finding of our series was a characteristic course of SRF resorption after surgery; the fovea attached first, and the SRF gradually resolved in the perifoveal area. Such a peculiar feature of foveal reattachment may be explained by the chronic detachment of the perifoveal macula and the viscous nature of the SRF.

The functional outcomes after the surgery varied and depended on many factors, such as the size of the $\mathrm{MH}$, height of the macular detachment, duration that the $\mathrm{MH}$ existed, degree of ischaemia and damage to the external layers after detachment. ${ }^{7}$ In general, patients with diabetic macular holes have a more limited and poorer visual prognosis after surgery than patients with idiopathic macular holes. In an earlier study by Brazitikos and Stangos, the visual acuity changed from 20/ 400 to $20 / 200$ in one eye and from $20 / 50$ to $20 / 40$ in the other eye. ${ }^{4}$ Flynn reported that the visual acuity changed from $20 /$ 400 to 20/200 in one eye and from 20/400 to 20/300 in the other eye. ${ }^{9}$ In a larger series by Yeh et al, the BCVA in LogMAR values improved from $1.75 \pm 0.18$ to $1.43 \pm 0.19$, with only 2 eyes out of 23 achieving a BCVA higher than $1.0 .^{8}$ Other authors have reported similar results. In the present study, visual acuity improved from preoperative $1.62 \pm 0.89$ LogMAR (range, 0.15-3.0 LogMAR) to $0.90 \pm 0.47$ LogMAR (range, 0.15-1.70) 6 months after the study.

In diabetic TRD patients undergoing surgery, OCT data, such as the ELM/EZ layers status, hyperreflective foci, and central foveal thickness, are important prognostic indicators for predicting and explaining final BCVA. ${ }^{11,12,19}$ Murakami et al found that disruption of the external limiting membrane, ellipsoid zone and hyperreflective foci were related to poor visual outcomes after vitrectomy. ${ }^{20}$ In our series, neither manual CFT $\left(\mathrm{r}_{\mathrm{s}}=-0.304, p=0.291\right)$ nor automated CFT $\left(r_{\mathrm{s}}=-0.420\right.$, $p=0.135$ ) was related to the final LogMAR BCVA. The most important predictive finding was ELM/EZ layers disruption, noted in $64.3 \%$ of cases with a significant negative correlation with the final LogMAR BCVA, $r_{\mathrm{b}}=-.0876, p=0.008$.

In summary, our findings show that the standard ILM peeling technique for full-thickness macular hole in eyes with active diabetic fibrovascular proliferation can help with the anatomical and functional restoration of the diabetic macula. Cases are frequently bilateral; close follow-up is important for unilateral cases. Residual subretinal fluid in the perifoveal area is detectable by OCT in cases of macular holes with diabetic tractional retinal detachment and usually resorbs gradually without any intervention. The retrospective nature of the study and the small sample size are limitations of this study. However, because of the rarity of macular holes associated with diabetic fibrovascular proliferation, it is nearly impossible to obtain large series within a single practice. Our observation supports previous reports concerning the beneficial anatomical and functional outcomes of vitrectomy with standard ILM peeling in patients with macular holes associated with diabetic fibrovascular proliferation.

\section{Disclosure}

There are no sources of any support for the work, received in the form of grants and/or equipment and drugs. There are not any competing financial interests in relation to the work described. The authors declare no conflicts of interest.

\section{References}

1. Mohamed Q, Gillies MC, Wong TY, Management of diabetic retinopathy: a systematic review. JAMA. 2007;298(8):902-916. doi:10.1001/ jama.298.8.902

2. Karimov MI, Gasymov EM, Aliyeva IJ, Akhundova LA, Rustambayova GR, Aliyev KD, An optical coherence tomography study of residual subfoveal fluid after successful pars plana vitrectomy in patients with diabetic tractional macular detachment. Eye (Lond). 2018;32(9):1472-1477. doi:10.1038/s41433-018-0111-6

3. Newman DK, Surgical management of the late complications of proliferative diabetic retinopathy. Eye (Lond). 2010;24(3):441-449. doi:10.1038/eye.2009.325

4. Brazitikos PD, Stangos NT, Macular hole formation in diabetic retinopathy: the role of coexisting macular edema. Doc Ophthalmol. 1999;97(3-4):273-278. doi:10.1023/A:1002117602501

5. Kurihara T, Noda K, Ishida S, Inoue M, Pars plana vitrectomy with internal limiting membrane removal for macular hole associated with proliferative diabetic retinopathy. Graefes Arch Clin Exp Ophthalmol. 2005;243(7):724-726. doi:10.1007/s00417-004-1081-x 
6. Amemiya T, Yoshida H. Macular hole in diabetic maculopathy. Ophthalmologica. 1978;177(4):188-191.

7. Lee BL, Vitrectomy for proliferative diabetic retinopathy and macular hole. Ann Ophthalmol. 2000;32(2):144-145. doi:10.1007/s12009000-0036-Z

8. Yeh PT, Cheng CK, Chen MS, Yang CH, Yang CM. Macular hole in proliferative diabetic retinopathy with fibrovascular proliferation. Retina. 2009;29(3):355-361.

9. Flynn HW, Macular hole surgery in patients with proliferative diabetic retinopathy. Arch Ophthalmol. 1994;112(7):877-878. doi:10.1001/archopht.1994.01090190021011

10. Lai CT, Yang CM. Development of macular holes in diabetic retinopathy with fibrovascular proliferation: report of four cases. Taiwan J Ophthalmol. 2015;5(2):85-89.

11. Chen SN, Yang CM. Internal limiting membrane flaps for coexistent macular hole and retinal detachment in eyes with proliferative diabetic retinopathy. $J$ Ophthalmol. 2018;3470731. Available from: https://doi.org/10.1155/2018/3470731.

12. Im JC, Kim JH, Park DH, Shin JP. Structural changes of the macula on optical coherence tomography after vitrectomy for proliferative diabetic retinopathy. Ophthalmologica. 2017;238(4):186-195. doi: $10.1159 / 000477826$

13. Liu L, Enkh-Amgalan I, Wang NK, et al. Results of macular hole surgery: evaluation based on the international vitreomacular traction study classification. Retina. 2018;38(5):900-906.

14. Ghoraba H, Types of macular holes encountered during diabetic vitrectomy. Retina. 2002;22(2):176-182. doi:10.1097/00006982200204000-00007
15. Felfeli T, Mandelcorn ED, Macular hole hydrodissection: surgical technique for the treatment of persistent, chronic, and large macular holes. Retina. 2019;39(4):743-752. doi:10.1097/IAE.000000 0000002013

16. Mason JO, Somaiya MD, White MF, Vail RS. Macular holes associated with diabetic tractional retinal detachments. Ophthalmic Surg Lasers Imaging. 2008;39(4):288-293. doi:10.3928/1542887720080701-12

17. Pessoa B, Dias DA, Baptista P, Coelho C, Beirão JNM, Meireles A. Vitrectomy outcomes in eyes with tractional diabetic macular edema Ophthalmic Res. 2019;61(2):94-99. doi:10.1159/000489459

18. Barzideh N, Johnson TM. Subfoveal fluid resolves slowly after pars plana vitrectomy for tractional retinal detachment secondary to proliferative diabetic retinopathy. Retina. 2007;27(6):740-743. doi:10.1097/IAE.0b013e318030c663

19. Dooley I, Laviers H, Papavasileiou E, McKechnie C, Zambarakji H. Spectral domain ocular coherence tomography findings pre-and post vitrectomy with fibrovascular membrane delamination for proliferative diabetic retinopathy. Eye (Lond). 2016;30(1):34-39. doi:10.1038/ eye. 2015.178

20. Murakami T, Uji A, Ogino K, et al. Macular morphologic findings on optical coherence tomography after microincision vitrectomy for proliferative diabetic retinopathy. Jpn $J$ Ophthalmol. 2015;59 (4):236-243. doi:10.1007/s10384-015-0382-4
Clinical Ophthalmology

\section{Publish your work in this journal}

Clinical Ophthalmology is an international, peer-reviewed journal covering all subspecialties within ophthalmology. Key topics include: Optometry; Visual science; Pharmacology and drug therapy in eye diseases; Basic Sciences; Primary and Secondary eye care; Patient Safety and Quality of Care Improvements. This journal is indexed on PubMed

\section{Dovepress}

Central and CAS, and is the official journal of The Society of Clinical Ophthalmology (SCO). The manuscript management system is completely online and includes a very quick and fair peer-review system, which is all easy to use. Visit http://www.dovepress.com/ testimonials.php to read real quotes from published authors. 